\title{
Drug transformation: Advances in drug delivery systems
}

Orlando Hung BSc (Pharmacy) MD FRCPC

S UCCESS stories often have a painful past. For decades, many pharmacologically active compounds have been discovered, isolated and synthesized. Unfortunately, many of these promising compounds did not advance beyond the laboratory bench to the clinical setting because of difficulties in finding a suitable delivery system. This is particularly true with highly lipophilic compounds, such as $\Delta^{9}$ - tetrahydrocannabinol (the active ingredient of cannabis), and other pharmacological agents with large molecular structures, such as peptides, proteins, genes, and nucleotides. In fact, propofol, probably the most commonly used iv anesthetic to date, had a somewhat tenuous introduction to clinical anesthesia.

Propofol, a highly lipophilic anesthetic agent with a $\log$ octanol-water partition coefficient of $4.33,{ }^{1}$ was developed and dissolved in Cremaphor EL for clinical trial in 1977. ${ }^{2}$ Unfortunately, Cremaphor EL had been shown to cause anaphylactic reactions, ${ }^{3,4}$ and propofol was subsequently withdrawn from development. ${ }^{2}$ Propofol was reformulated in a soybean macroemulsion in mid-1980 and was used clinically in the United Kingdom and New Zealand in 1986 and in the United States in 1989.5,6 The introduction of this new propofol formulation has transformed propofol into one of the most important iv anesthetics ever employed. The development of a suitable formulation for propofol exemplifies how drug delivery systems can play a significant role in modern therapeutics.

Over the past several decades, significant advances in the development of delivery systems have taken place to meet the challenges associated with the unique physicochemical properties of various pharmacological agents. New drug carrier technologies, such as colloidal drug carriers (liposomes and nanoparticles), have been used to modify the delivery of large molecules or molecules with exceptional lipophilic- ity. ${ }^{7}$ Colloidal drug carriers are particularly useful in the delivery of peptides, genes, and oligonucleotides because they can provide pharmacologically-active agent protection from degradation in biological fluids and they can promote the penetration of active substances into cells.

Liposomes are microscopic phospholipid-bilayered vesicles that are biocompatible, biodegradable and nonimmumnogenic. They can be used to entrap both hydrophilic and lipophilic drug molecules, ${ }^{8}$ making them highly versatile as drug delivery systems. In this context, the rate of drug release by the liposome into the local environment is primarily determined by the physicochemical properties of the liposome. Liposomes can be tailored by modification of their size, layers (unilamellar, or multilamellar), composition, and surface charge to provide a controlled and sustained drug release system.

In this issue of the Journal, using a rat infraorbital nerve blockade technique to evaluate anesthetic effect, Cereda et al. report that liposomes can be used to provide effective drug-delivery systems for prilocaine, lidocaine and mepivacaine. ${ }^{9}$ Their findings suggest that encapsulated formulations can induce an increase in the intensity of anesthetic effect, and time to recovery when compared to plain local anesthetic solutions. They conclude that liposome encapsulation can be used to increase both the anesthetic duration and intensity.

The use of liposomes to encapsulate drugs is not new. Liposomal lidocaine (ELA-Max Topical Anesthetic) has been commercially available for several years in the United States and offers a more rapid onset and less expensive alternative to the eutectic mixture of local anesthetic (EMLA) formulations. ${ }^{10}$ Many pharmaceutical companies conduct studies on encapsulation of macromolecules, such as peptides, proteins and nucleic acids. These macromolecules are

From the Departments of Anesthesia, Surgery and Pharmacology, Dalhousie University, Queen Elizabeth II Health Sciences Center, Halifax, Nova Scotia, Canada.

Address correspondence to: Dr. Orlando Hung, Departments of Anesthesia, Surgery and Pharmacology, Dalhousie University, Queen Elizabeth II Health Sciences Center, 1278 Tower Rd., Halifax, Nova Scotia B3H 2Y9, Canada. Phone: 902-473-7767; Fax: 902-473-4493; E-mail: hungorla@dal.ca

Disclosure statement: Dr. Orlando Hung is the owner of the U.S. Patent of "Pain Management with liposome-encapsulated analgesic drugs" and has filed several other patent applications for drug delivery systems. 
protected from in vivo hydrolysis by encapsulation. In addition to sustained drug release, liposomes have also been shown to improve the therapeutic index of drugs by increasing their efficacy and reducing their toxicity. This has been notably the case for chemotherapeutic agents (Doxil and Caelyx - liposomal doxorubicin) ${ }^{11}$ and antimicrobials (Ambisome $\mathrm{TM}^{\mathrm{TM}}$ - Amphotericin B). ${ }^{12,13}$ Although these liposomal preparations have been shown to be effective and safe, the bioavailability of iv liposomal chemotherapeutic agents is reduced by the rapid uptake of liposomes by the reticuloendothelial system. To minimize rapid uptake of liposomes by the reticuloendothelial system and to improve their bioavailability, a novel pegylated liposomal system coated with polyethylene glycol ("stealth liposomes") have been developed, ${ }^{11,14,15}$ thus improving drug delivery to the tumour while decreasing toxicity to other cells.

More recently, substantial interest has been shown in developing drug delivery systems utilizing nanoparticles, micro-particles composed of biodegradable polymers. ${ }^{16,17}$ Like liposomes, nanoparticles have been developed as an important strategy to deliver drugs, proteins, vaccines and nucleotides. However, they have some advantages over liposomes in terms of stability both during storage and in vivo. ${ }^{7}$ They may consist of either a polymeric matrix (nanospheres) or a reservoir system in which an oily or aqueous medium is surrounded by a polymeric wall (nanocapsules). Nanoparticles have been shown to modify release, pharmacokinetics, and body distribution of wellestablished drugs. ${ }^{18}$

So why should we, as anesthesiologists, care about these advances in drug delivery systems? Simply because these advances will likely change the future of drug development. Instead of searching only for newer drug molecules, it is possible to modify the pharmacokinetic and pharmacodynamic properties of existing drugs by manipulating different drug delivery systems. As Rosen and Abribat have suggested, it is now possible to "make old drugs new" by utilizing these advanced drug delivery technologies. ${ }^{19}$ Wellestablished drugs with proven efficacy and safety can be transformed into new entities with modified and enhanced clinical applications by manipulating the formulation of the drug delivery systems.

We are beginning to see evidence of this transformation of "old into new again" in the practice of anesthesia. Recently, the Food and Drug Administration of the United States approved the use of epidural administration of liposomal morphine (DepoDur) for prolonged postoperative analgesia with up to $48 \mathrm{hr}$ of relief after a single injection. ${ }^{20}$ Clinical trials of the pulmonary administration of liposomal fentanyl (AeroLEF ${ }^{\mathrm{TM}}$ ) for rapid onset and long duration of analgesia are currently being conducted for patients suffering from postoperative and cancer pain. $^{21}$ Recently, Morey and colleagues reported the successful preparation of propofol nanoparticles (microemulsion) which has the potential to reduce the risk of bacterial contamination associated with soybean oil-based macroemulsions of propofol. ${ }^{22}$ These are exciting developments for anesthesiologists and we should embrace this new knowledge and apply it to our clinical practice.

While these developments in drug delivery look promising, the road toward transforming well-established drugs may be a long and winding one. There are limitations to these drug delivery systems: shelf life, aggregation, leakage, and toxicity of polymers - to name a few. However, with rigorous and thorough investigation and a commitment to the highest standard of safety, innovation, and collaboration, we might one day look back on our current clinical practice and marvel at the changes that have been brought about by this exciting research.

\section{La transformation des médicaments par de nouveaux vecteurs}

L'histoire des réussites a souvent un passé douloureux. Pendant des décennies, des composés pharmacologiques actifs ont été découverts, isolés et synthétisés. Mais nombre de ces mélanges prometteurs ne sont pas allés plus loin que la table de laboratoire à cause des difficultés à trouver un mode d'administration approprié. C'est particulièrement vrai des composés hautement lipophiles, comme le $\Delta^{9}$ - tétrahydrocannabinol, principe actif du cannabis, et d'autres agents pharmacologiques comportant de grandes structures moléculaires comme les peptides, les protéines, les gènes et les nucléotides. De fait, le propofol, l'anesthésique iv sans doute le plus couramment utilisé jusqu’à présent, a connu des débuts plutôt précaires en anesthésie clinique.

Agent anesthésique hautement lipophile, le propofol présente un coefficient de partage octanol-eau de $4,33 .{ }^{1}$ Il a été mis au point et dissous dans le Cremaphor EL pour essai clinique en $1977 .{ }^{2}$ Mais on a découvert que le Cremaphor EL causait des réac- 
tions anaphylactiques ${ }^{3,4}$ et le propofol a été retiré. ${ }^{2}$ Reformulé dans une macroémulsion de soya au milieu des années 1980, il a été utilisé en clinique au Royaume-Uni et en Nouvelle-Zélande en 1986 et aux États-Unis en 1989.5,6 L'introduction de cette nouvelle préparation de propofol en a fait l'un des plus importants anesthésiques iv jamais employés et montre comment les systèmes de vecteurs peuvent jouer un rôle significatif dans les thérapies modernes.

Au cours des dernières décennies, des progrès significatifs ont été réalisés dans le développement des systèmes de vecteurs pour faire face aux défis associés aux propriétés physico-chimiques uniques de différents agents pharmacologiques. De nouveaux vecteurs colloïdaux (liposomes et nanoparticules), ont été utilisés pour modifier le transport de grosses molécules ou de molécules dotées de lipophilie exceptionnelle. ${ }^{7}$ Ces vecteurs sont utiles en particulier comme transporteur des peptides, des gènes et des oligonucléotides, car ils peuvent protéger les agents pharmacologiques actifs de la dégradation dans les liquides biologiques et favoriser la pénétration intracellulaire des substances actives.

Les liposomes sont des vésicules microscopiques de bicouches de phospholipides biocompatibles, biodégradables et non immunogènes. Ils peuvent piéger des molécules médicamenteuses autant hydrophiles que lipophiles, ${ }^{8}$ ce qui en fait des vecteurs très polyvalents. Dans ce contexte, le taux de libération du médicament par le liposome dans l'environnement local est principalement déterminé par les propriétés physicochimiques du liposome. Les liposomes sont adaptables par une modification de leur taille, de leurs couches (unilamellaire ou multilamellaire), de leur composition et de leur charge superficielle pour maintenir la libération contrôlée et prolongée des médicaments.

Dans le présent numéro du Journal, Cereda et coll., après avoir utilisé une technique de blocage du nerf infra-orbitaire chez le rat, rapportent que les liposomes peuvent assurer un système efficace d'administration de la prilocaïne, de la lidocaïne et de la mépivacaïne. ${ }^{9}$ Leurs résultats montrent que les préparations encapsulées peuvent augmenter l'intensité de l'effet anesthésique et le temps de récupération en comparaison avec les solutions d'anesthésiques locaux. Ils concluent que l'encapsulation dans des liposomes peut servir à accroître la durée et l'intensité de l'anesthésique.

L'emploi des liposomes pour encapsuler les médicaments n'est pas nouveau. La lidocaïne liposomique (ELA-Max Topical Anesthetic) est commercialement disponible depuis quelques années aux États-Unis et présente un début d'action plus rapide. Elle peut remplacer, à meilleur prix, le mélange eutectique de préparations d'anesthésiques locaux (EMLA). ${ }^{10}$ De nombreuses compagnies pharmaceutiques ont menédes études sur l'encapsulation de macromolécules, comme les peptides, les protéines et les acides nucléiques. Ces macromolécules sont protégées de l'hydrolyse in vivo par l'encapsulation. En plus d'entretenir la libération du médicament, les liposomes peuvent en améliorer l'index thérapeutique en augmentant leur efficacité et en réduisant leur toxicité. Cela a été notamment le cas des agents chimiothérapeutiques (Doxil et Caelyx - doxorubicin liposomique $)^{11}$ et des antimicrobiens (Ambisome ${ }^{\mathrm{TM}}$ - Amphotéricin B). ${ }^{12,13}$ Même si ces préparations liposomiques se sont montrées efficaces et sûres, la biodisponibilité des agents chimiothérapeutiques liposomiques $i v$ est réduite par le captage rapide des liposomes par le système réticulo-endothélial. Pour minimiser ce captage et améliorer la biodisponibilité, un nouveau système liposomique pégylé enrobé de polyéthylène glycol («liposomes furtifs») a été élaboré, ${ }^{11,14,15}$ améliorant ainsi le transport des médicaments vers la tumeur tout en diminuant la toxicité pour les autres cellules.

Plus récemment, il y a eu un grand intérêt pour le développement de vecteurs de médicaments qui utilisent des nanoparticules, des microparticules composées de polymères biodébradables. ${ }^{16,17}$ Les nanoparticules ont été conçues, comme les liposomes, pour l'administration des médicaments, des protéines, des vaccins et des nucléotides. Or, ils ont certains avantages sur les liposomes quant à leur stabilité pendant le stockage et in vivo. ${ }^{7}$ Ils peuvent consister soit en une matrice polymérique (nanosphères), soit en un système réservoir dans lequel un milieu huileux ou aqueux est entouré d'une membrane polymérique (nanocapsules).Les nanoparticules modifient la libération, la pharmacocinétique et la distribution dans l'organisme de médicaments bien établis. ${ }^{18}$

Alors, pourquoi devrions-nous nous intéresser à ces nouveaux vecteurs de médicaments ? Tout simplement parce que ces progrès vont sans doute changer le développement des médicaments. Au lieu de chercher seulement de nouvelles molécules, il sera possible de modifier les propriétés pharmacocinétiques et pharmacodynamiques des médicaments existants en manipulant différents systèmes de vecteurs. Comme le suggèrent Rosen et Abribat, nous pouvons maintenant «faire du neuf avec du vieux» en utilisant les nouvelles technologies des vecteurs de médicaments. ${ }^{19}$ Les médicaments bien connus, prouvés efficaces et sûrs, peuvent être ainsi transformés en de nouvelles entités aux applications cliniques modifiées et améliorées par l'adaptation de la composition des systèmes de transporteurs. 
Nous commençons à voir la preuve de ce «recyclage» de médicaments dans la pratique de l'anesthésie. Récemment, la Food and Drug Administration des États-Unis a approuvé l'usage de l'administration péridurale de la morphine liposomique (DepoDur) pour l'analgésie postopératoire prolongée jusqu'à 48 $\mathrm{h}$ après une seule injection. ${ }^{20}$ Des essais cliniques sur l'inhalation de fentanyl liposomique (AeroLEF ${ }^{\mathrm{TM}}$ ), pour une début d'action rapide et une analgésie de longue durée, sont présentement en cours auprès de patients souffrant de douleur postopératoire et cancéreuse. ${ }^{21}$ Dernièrement, Morey et ses collègues ont présenté la préparation réussie de nanoparticules de propofol (microémulsion) ayant le potentiel de réduire le risque de contamination bactérienne associée aux macroémulsions de propofol à base d'huile de soya. ${ }^{22}$ Ce sont des réalisations passionnantes pour les anesthésiologistes et nous devrions adopter ces nouvelles connaissances et les appliquer à notre pratique clinique.

Bien que ces progrès semblent prometteurs, la route vers la transformation des médicaments bien établis pourrait être longue et sinueuse. Ces vecteurs ont des limites: la durée de conservation, l'agrégation, les fuites et la toxicité des polymères, pour n'en nommer que quelque-unes. Cependant, avec des recherches rigoureuses et approfondies selon les plus hauts standards de sécurité, d'innovation et de collaboration, nous pourrions un jour regarder en arrière et nous émerveiller des changements apportés par cette stimulante recherche.

\section{References}

1 Dutta S, Ebling WF. Formulation-dependent brain and lung distribution kinetics of propofol in rats. Anesthesiology 1998; 89: 678-85.

2 Baker MT, Naguib M. Propofol: the challenges of formulation. Anesthesiology 2005; 103: 860-76.

3 Dye D, Watkins J. Suspected anaphylactic reaction to Cremophor EL. Br Med J 1980; 280: 1353.

4 Huttel MS, Schou Olesen A, Stoffersen E. Complementmediated reactions to diazepam with Cremophor as solvent (Stesolid MR). Br J Anaesth 1980; 52: 77-90.

5 Dutta S, Matsumoto $\Upsilon$, Ebling WF. Propofol pharmacokinetics and pharmacodynamics assessed from a cremophor EL formulation. J Pharm Sci 1997; 86: 967-9.

6 Thompson KA, Goodale DB. The recent development of propofol (DIPRIVAN). Intensive Care Med 2000; 26(suppl 4): S400-4.

7 Barratt G. Colloidal drug carriers: achievements and perspectives. Cell Mol Life Sci 2003; 60: 21-37.

8 Bangham AD, Standish MM, Watkins JC. Diffusion of univalent ions across the lamellae of swollen phospho- lipids. J Mol Biol 1965; 13: 238-52.

9 Cereda CM, Bruschini G, de Araújo DR, de Paula E. Liposomal formulations of prilocaine, lidocaine and mepivacaine prolong analgesic duration. Can J Anesth 2006; 53: 1092-7.

10 Eidelman A, Weiss JM, Lau J, Carr DB. Topical anesthetics for dermal instrumentation: a systematic review of randomized, controlled trials. Ann Emerg Med 2005; 46: 343-51.

11 Rose PG. Pegylated liposomal doxorubicin: optimizing the dosing schedule in ovarian cancer. Oncologist 2005; 10: 205-14.

12 Kshirsagar NA, Pandya SK, Kirodian GB, Sanath S. Liposomal drug delivery system from laboratory to clinic. J Postgrad Med 2005; 51 Suppl 1: S5-15.

13 Meyerhoff A. U.S. Food and Drug Administration approval of AmBisome (liposomal amphotericin B) for treatment of visceral leishmaniasis. Clin Infect Dis 1999; 28: 42-8; discussion 49-51.

14 Harris JM, Martin NE, Modi M. Pegylation: a novel process for modifying pharmacokinetics. Clin Pharmacokinet 2001; 40: 539-51.

15 Reddy KR. Controlled-release, pegylation, liposomal formulations: new mechanisms in the delivery of injectable drugs. Ann Pharmacother 2000; 34: 915-23.

16 Emerich DF, Thanos CG. Nanotechnology and medicine. Expert Opin Biol Ther 2003; 3: 655-63.

17 Gordon EM, Hall FL. Nanotechnology blooms, at last (Review). Oncol Rep 2005; 13: 1003-7.

18 Kayser O, Lemke A, Hernandez-Trejo N. The impact of nanobiotechnology on the development of new drug delivery systems. Curr Pharm Biotechnol 2005; 6: 3-5.

19 Rosen H, Abribat T. The rise and rise of drug delivery. Nat Rev Drug Discov 2005; 4: 381-5.

20 Viscusi ER. Emerging techniques for postoperative analgesia in orthopedic surgery. Am J Orthop 2004; 33: 13-6.

21 Chan V, Valle-Leutri F, Clark J, Rossiter-Rooney M. Self-titrated aerosolized liposome-encapsulated fentanyl results in rapid onset and sustained analgesia following ACL knee surgery. Anesthesiology 2004; 101: A77 (abstract).

22 Morey TE, Modell JH, Shekhawat D, et al. Preparation and anesthetic properties of propofol microemulsions in rats. Anesthesiology 2006; 104: 1184-90. 